\title{
Plant-based insect repellents: a review of their efficacy, development and testing
}

Marta Ferreira Maia ${ }^{1,2}$, Sarah J Moore $e^{1,2^{*}}$

\begin{abstract}
Plant-based repellents have been used for generations in traditional practice as a personal protection measure against host-seeking mosquitoes. Knowledge on traditional repellent plants obtained through ethnobotanical studies is a valuable resource for the development of new natural products. Recently, commercial repellent products containing plant-based ingredients have gained increasing popularity among consumers, as these are commonly perceived as "safe" in comparison to long-established synthetic repellents although this is sometimes a misconception. To date insufficient studies have followed standard WHO Pesticide Evaluation Scheme guidelines for repellent testing. There is a need for further standardized studies in order to better evaluate repellent compounds and develop new products that offer high repellency as well as good consumer safety. This paper presents a summary of recent information on testing, efficacy and safety of plant-based repellents as well as promising new developments in the field.
\end{abstract}

\section{Background}

Most plants contain compounds that they use in preventing attack from phytophagous (plant eating) insects. These chemicals fall into several categories, including repellents, feeding deterrents, toxins, and growth regulators. Most can be grouped into five major chemical categories: (1) nitrogen compounds (primarily alkaloids), (2) terpenoids, (3) phenolics, (4) proteinase inhibitors, and (5) growth regulators. Although the primary functions of these compounds is defence against phytophagous insects, many are also effective against mosquitoes and other biting Diptera, especially those volatile components released as a consequence of herbivory [1]. The fact that several of these compounds are repellent to haematophagous insects could be an evolutionary relict from a plant-feeding ancestor, as many of these compounds evolved as repellents to phytophagous insects [2], and this repellent response to potentially toxic compounds is well conserved in the lineage of Diptera (True Flies). Insects detect odours when that volatile odour binds to odorant receptor (OR) proteins displayed on ciliated dendrites of specialized odour receptor neurons (ORNs) that are exposed to the external environment,

\footnotetext{
* Correspondence: sarah.moore@lshtm.ac.uk

'Disease Control Department, London School of Hygiene and Tropical

Medicine, Keppel Street, London WC1E 7HT, UK

Full list of author information is available at the end of the article
}

often on the antennae and maxillary palps of the insect, and some ORNs, such as OR83b that is important in olfaction and blocked by the gold-standard synthetic repellent DEET (N, N-diethyl-3-methylbenzamide) [3], are highly conserved across insect species $[4,5]$. Plants commonly produce volatile "green leaf volatiles" when leaves are damaged in order to deter herbivores [6], and several authors have shown strong responses of mosquito odour receptors to this class of volatiles including geranyl acetate and citronellal [7], 6-methyl-5- hepten2 -one and geranylacetone [8]. Interestingly, the same odour receptors that respond to DEET also respond to thujone eucalyptol and linalool in Culex quinquefasciatus[9]. In Anopheles gambiae, the DEET receptor OR83b is stimulated by citronellal, but is also modulated by the TRPA1 cation channel [10]. However, it is most likely that many plant volatiles are deterrent or repellent because they have high vapour toxicity to insects $[11,12]$.

This repellency of plant material has been exploited for thousands of years by man, most simply by hanging bruised plants in houses, a practice that is still in wide use throughout the developing countries [13]. Plants have also been used for centuries in the form of crude fumigants where plants were burnt to drive away nuisance mosquitoes and later as oil formulations applied to the skin or clothes which was first recorded in 
writings by ancient Greek [14], Roman [15] and Indian scholars [16] (Figure 1). Plant-based repellents are still extensively used in this traditional way throughout rural communities in the tropics because for many of the poorest communities the only means of protection from mosquito bites that are available [13], and indeed for some of these communities [17], as in the Europe and North America [18] "natural" smelling repellents are preferred because plants are perceived as a safe and trusted means of mosquito bite prevention.

The discovery of new plant-based repellents is heavily reliant on ethnobotany. This is the targeted search for medicinal plants through in-depth interviews with key informants knowledgeable in folk-lore and traditional medicine. It is common practice to conduct ethnobotanical surveys using structured interviews, combined with

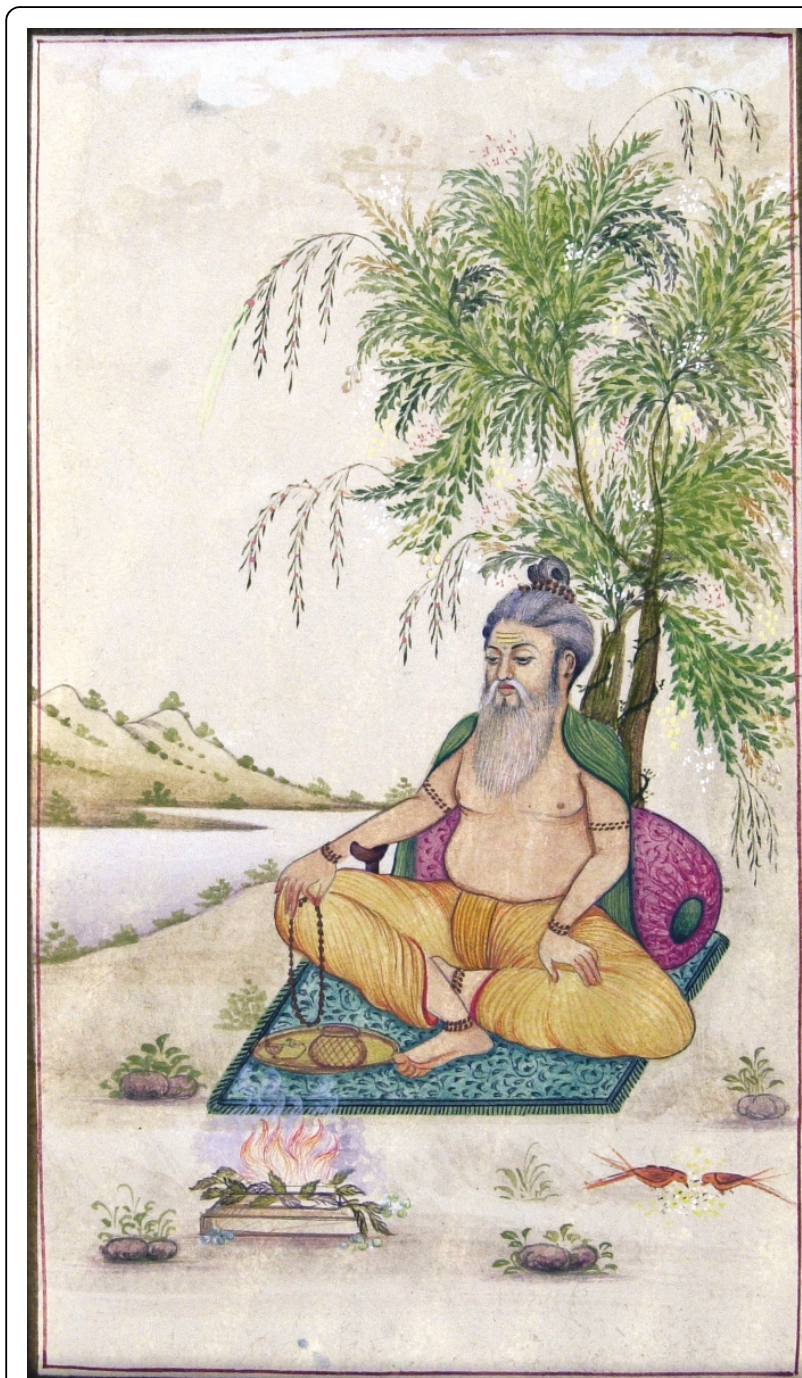

Figure 1 Moghul painting illustrating a man burning neem leaves near a river where biting insects would be present $\left({ }^{\odot}\right.$ Dr Sarah Moore) the collection of plant voucher Specimens (Figure 2), to evaluate plant use by indigenous ethnic groups [19]. Questions are asked about plant usage, abundance and source. This is a more direct method of identifying plants with a potential use than general screening of all plants in an area. A second means is bio-prospecting, where plants are systematically screened for a particular mode of action, which is a costly and labour intensive means of identifying new repellents. However, mass screening of plants for repellent activity was the way by which PMD (para-methane 3-8, diol), an effective and commercially available repellent was discovered in the 1960s [20].

\section{PMD from lemon eucalyptus (Corymbia citriodora) extract}

Corymbia citriodora (Myrtaceae), also known as lemon eucalyptus, is a potent natural repellent extracted from the leaves of lemon eucalyptus trees (Table 1). It was discovered in the 1960s during mass screenings of plants used in Chinese traditional medicine. Lemon eucalyptus essential oil, comprising $85 \%$ citronellal, is used by cosmetic industries due to its fresh smell [21]. However, it was discovered that the waste distillate remaining after hydro-distillation of the essential oil was far more effective at repelling mosquitoes than the essential oil itself. Many plant extracts and oils repel mosquitoes, with their effect lasting from several minutes to several hours (Table 1). Their active ingredients tend to be highly volatile, so although they are effective repellents for a short period after application, they rapidly evaporate leaving the user unprotected. The exception to this is para-menthane 3, 8 diol, which has a lower vapour pressure than volatile monoterpines found in most plant oils [22] and provides very high protection from a broad range of insect vectors over several hours [23], whereas the essential oil is repellent for around one hour [24]. PMD is the only plant-based repellent that has been advocated for use in disease endemic areas by the CDC (Centres for Disease Control) [25], due to its proven clinical efficacy to prevent malaria [26] and is considered to pose no risk to human health [27]. It should be noted that the essential oil of lemon eucalyptus does not have EPA (Environmental Protection Agency) registration for use as an insect repellent.

\section{Citronella}

Essential oils and extracts belonging to plants in the citronella genus (Poaceae) are commonly used as ingredients of plant-based mosquito repellents (Table 1), mainly Cymbopogon nardus that is sold in Europe and North America in commercial preparations. Citronella has found its way into many commercial preparations through its familiarity, rather than its efficacy. Citronella 


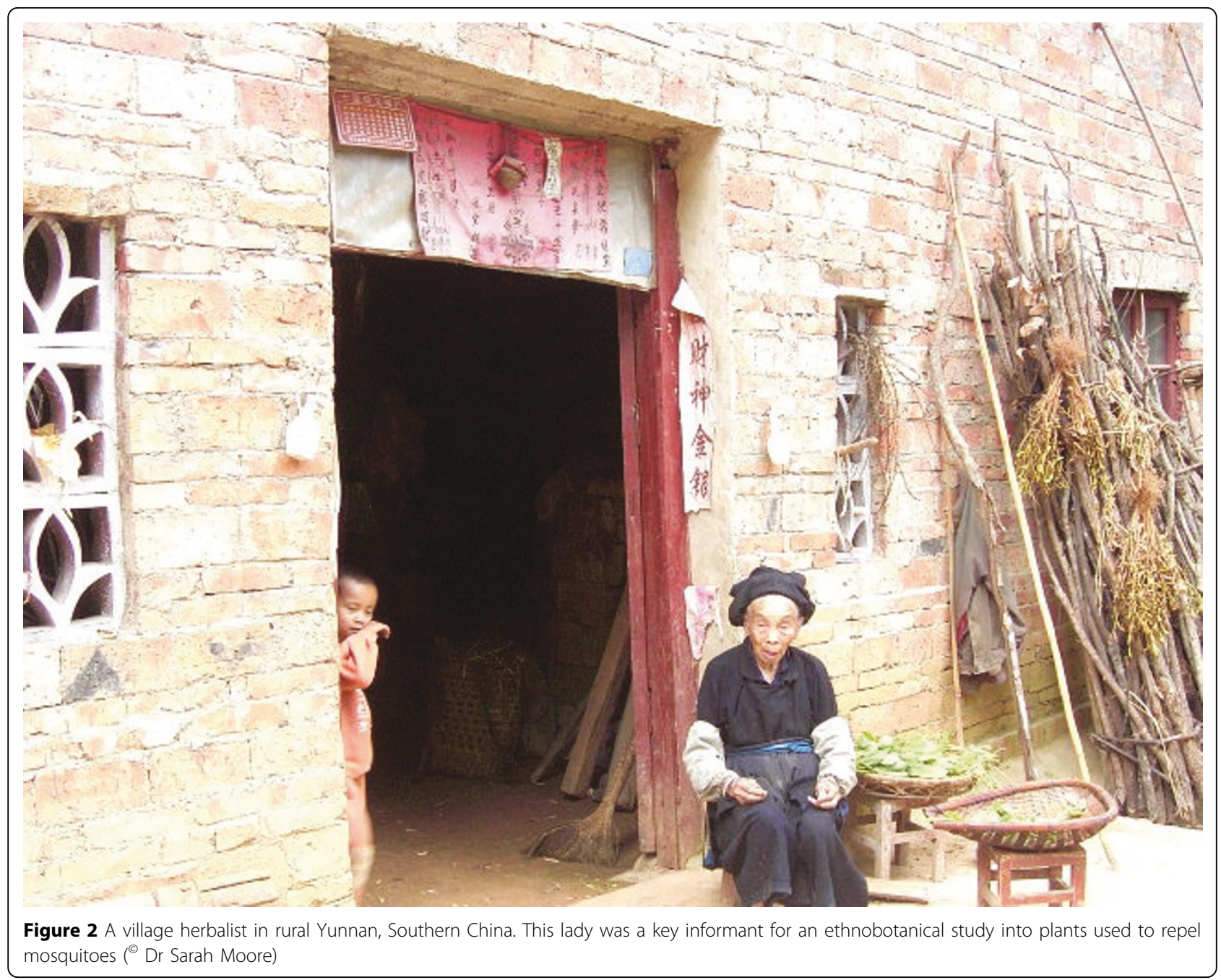

was originally extracted for use in perfumery, and its name derives from the French citronelle around 1858 [28]. It was used by the Indian Army to repel mosquitoes at the beginning of the $20^{\text {th }}$ century [29] and was then registered for commercial use in the USA in 1948 [30]. Today, citronella is one of the most widely used natural repellents on the market, used at concentrations of $5-10 \%$. This is lower than most other commercial repellents but higher concentrations can cause skin sensitivity. However, there are relatively few studies that have been carried out to determine the efficacy of essential oils from citronella as arthropod repellents. Citronella-based repellents only protect from host-seeking mosquitoes for about two hours although formulation of the repellent is very important $[31,32]$. Initially, citronella, which contains citronellal, citronellol, geraniol, citral, $\alpha$ pinene, and limonene, is as effective dose for dose as DEET [33], but the oils rapidly evaporate causing loss of efficacy and leaving the user unprotected. However, by mixing the essential oil of Cymbopogon winterianus with a large molecule like vanillin $(5 \%)$ protection time can be considerable prolonged by reducing the release rate of the volatile oil [34]. Recently, the use of nanotechnology has allowed slower release rates of oils to be achieved, thus prolonging protection time [35]. Encapsulated citronella oil nanoemulsion is prepared by high-pressure homogenization of $2.5 \%$ surfactant and $100 \%$ glycerol, to create stable droplets that increase the retention of the oil and slow down release. The release rate relates well to the protection time so that a decrease in release rate can prolong mosquito protection time [35]. Another means of prolonging the effect of natural repellents is microencapsulation using gelatin-arabic gum microcapsules, which maintained the repellency of citronella up to 30 days on treated fabric stored at room temperature $\left(22^{\circ} \mathrm{C}\right)$ [36]. The use of these technologies to enhance the performance of natural repellents may revolutionize the repellent market and make plant oils a more viable option for use in long-lasting repellents. However, for the time-being 
Table 1 An overview of repellent plant efficacy from literature review

\begin{tabular}{|c|c|c|c|c|c|c|c|}
\hline Plant & Location & $\begin{array}{l}\text { Other } \\
\text { names }\end{array}$ & $\begin{array}{l}\text { Repellent } \\
\text { compound(s) }\end{array}$ & Tested mode of use & Repellency \% protection & $\begin{array}{l}\text { Study } \\
\text { type }\end{array}$ & Ref \\
\hline \multicolumn{8}{|l|}{ MYRTACEAE } \\
\hline \multirow[t]{10}{*}{ Corymbia citriodora } & \multirow{10}{*}{$\begin{array}{l}\text { Australia } \\
\text { Brazil } \\
\text { Bolivia } \\
\text { China } \\
\text { India } \\
\text { Ethiopia } \\
\text { Tanzania } \\
\text { Kenya }\end{array}$} & \multirow{10}{*}{$\begin{array}{l}\text { lemon } \\
\text { eucalyptus } \\
\text { lemon } \\
\text { scented } \\
\text { gum } \\
\text { quwenling }\end{array}$} & \multirow{10}{*}{$\begin{array}{l}\text { citronellal } \\
\text { PMD (by } \\
\text { product of } \\
\text { hidrodistillation) } \\
\text { (p-menthane- } \\
\text { 3,8-diol) } \\
\text { citronellol } \\
\text { limonene } \\
\text { geraniol } \\
\text { isopulegol } \\
\delta \text {-pinene }\end{array}$} & $\begin{array}{l}30 \% \text { PMD applied } \\
\text { topically }\end{array}$ & $\begin{array}{l}96.88 \% \text { protection from } \\
\text { mosquitoes for } 4 \text { hours }\end{array}$ & $\begin{array}{l}\text { field study } \\
\text { in Bolivia }\end{array}$ & {$[45]$} \\
\hline & & & & $\begin{array}{l}\text { PMD towelette } \\
(0.575 \mathrm{~g}) \text { applied } \\
\text { topically }\end{array}$ & $\begin{array}{l}90 \% \text { protection from } A n . \\
\text { arabiensis for } 6 \text { hours }\end{array}$ & $\begin{array}{l}\text { laboratory } \\
\text { study }\end{array}$ & [112] \\
\hline & & & & $\begin{array}{l}\text { 50\% PMD applied } \\
\text { topically }\end{array}$ & $\begin{array}{l}\text { 100\% protection from An } \\
\text { gambiae and An. funestus for } \\
6-7 \text { hours }\end{array}$ & $\begin{array}{l}\text { field study } \\
\text { in Tanzania }\end{array}$ & [113] \\
\hline & & & & $\begin{array}{l}20 \% \text { PMD }(1.7 \mathrm{mg} / \\
\left.\mathrm{cm}^{2}\right) \text { applied topically }\end{array}$ & $\begin{array}{l}100 \% \text { protection for } 11-12 \\
\text { hours against A. stephensi }\end{array}$ & $\begin{array}{l}\text { laboratory } \\
\text { study }\end{array}$ & {$[64]$} \\
\hline & & & & $\begin{array}{l}20 \% \text { PMD applied } \\
\text { topically }\end{array}$ & $\begin{array}{l}100 \% \text { protection against } A e \text {. } \\
\text { Aegypti for } 120 \text { minutes }\end{array}$ & $\begin{array}{l}\text { Laboratory } \\
\text { study }\end{array}$ & {$[52]$} \\
\hline & & & & $\begin{array}{l}\text { thermal expulsion } \\
\text { (leaves) }\end{array}$ & $\begin{array}{l}78.7 \% \text { protection from } A n . \\
\text { arabiensis } \\
76.8 \% \text { protection from } A n . \\
\text { pharaoensis }\end{array}$ & $\begin{array}{l}\text { field study } \\
\text { in Ethiopia }\end{array}$ & [114] \\
\hline & & & & direct burning (leaves) & $\begin{array}{l}70.1 \% \text { protection from } A n . \\
\text { arabiensis } \\
72.9 \% \text { protection from } A n . \\
\text { pharaoensis }\end{array}$ & $\begin{array}{l}\text { field study } \\
\text { in Ethiopia }\end{array}$ & [114] \\
\hline & & & & $\begin{array}{l}\text { periodic thermal } \\
\text { expulsion (leaves) }\end{array}$ & $\begin{array}{l}\text { 74.5\% protection from An. } \\
\text { gambiae s.s. }\end{array}$ & $\begin{array}{l}\text { semi-field } \\
\text { study in } \\
\text { Kenya }\end{array}$ & {$[62]$} \\
\hline & & & & $\begin{array}{l}\text { periodic direct burning } \\
\text { (leaves) }\end{array}$ & $\begin{array}{l}51.3 \% \text { protection from } A n \text {. } \\
\text { gambiae s.s. }\end{array}$ & $\begin{array}{l}\text { semi-field } \\
\text { study in } \\
\text { Kenya }\end{array}$ & {$[62]$} \\
\hline & & & & $\begin{array}{l}\text { thermal expulsion } \\
\text { (leaves) }\end{array}$ & $\begin{array}{l}48.71 \% \text { protection from } A n . \\
\text { gambiae s.l. }\end{array}$ & $\begin{array}{l}\text { field study } \\
\text { in Kenya }\end{array}$ & [115] \\
\hline Eucalyptus spp. & $\begin{array}{l}\text { Guinea-Bissau } \\
\text { Ethiopia } \\
\text { Tanzania } \\
\text { Portugal }\end{array}$ & eucalyptus & $\begin{array}{l}\text { 1,8-cineole } \\
\text { citronellal } \\
\text { Z- and } \alpha \text { - citral } \\
\alpha \text {-pinene }\end{array}$ & $\begin{array}{l}\text { thermal expulsion } \\
\text { (leaves) }\end{array}$ & $\begin{array}{l}72.2 \% \text { protection from } \\
\text { mosquitoes for } 2 \text { hours }\end{array}$ & $\begin{array}{l}\text { field study } \\
\text { in Guinea } \\
\text { Bissau }\end{array}$ & [116] \\
\hline \multirow[t]{2}{*}{ E. camaldulensis } & Ethiopia & & & $\begin{array}{l}\text { thermal expulsion } \\
\text { (leaves) }\end{array}$ & $\begin{array}{l}71.9 \% \text { protection from } A n . \\
\text { arabiensis } \\
72.2 \% \text { protection from } A n . \\
\text { pharaoensis }\end{array}$ & $\begin{array}{l}\text { field study } \\
\text { in Ethiopia }\end{array}$ & [114] \\
\hline & & & & direct burning (leaves) & $\begin{array}{l}65.3 \% \text { protection from } A n . \\
\text { arabiensis } \\
66.6 \% \text { protection from } A n . \\
\text { pharaoensis }\end{array}$ & $\begin{array}{l}\text { field study } \\
\text { in Ethiopia }\end{array}$ & [114] \\
\hline \multirow[t]{2}{*}{$\begin{array}{l}\text { Eugenia caryophyllus or } \\
\text { Syzygium aromaticum } \\
\text { or Eugenia aromaticu }\end{array}$} & India & $\begin{array}{l}\text { clove } \\
\text { lavang } \\
\text { cravinho- } \\
\text { da-india }\end{array}$ & $\begin{array}{l}\text { Eugenol } \\
\text { carvacrol } \\
\text { thymol } \\
\text { cinnamaldehyde }\end{array}$ & $\begin{array}{l}100 \% \text { essential oil } \\
\text { applied topically }\end{array}$ & $\begin{array}{l}100 \% \text { protection against } A e . \\
\text { aegypti for } 225 \text { minutes } \\
100 \% \text { protection against } A n . \\
\text { albimanus for } 213 \text { minutes }\end{array}$ & $\begin{array}{l}\text { laboratory } \\
\text { study }\end{array}$ & {$[65]$} \\
\hline & & & & $\begin{array}{l}100 \% \text { essential oil } \\
\text { applied topically }\end{array}$ & $\begin{array}{l}\text { 100\% protection against } A e . \\
\text { aegypti for } 120 \text { min. } \\
100 \% \text { protection against } C . \\
\text { quinquefasciatus for } 240 \text { min. } \\
100 \% \text { protection against } A n . \\
\text { dirus for } 210 \text { min. }\end{array}$ & $\begin{array}{l}\text { laboratory } \\
\text { study }\end{array}$ & {$[31]$} \\
\hline \multicolumn{8}{|l|}{ VERBENACEAE } \\
\hline Lippia spp. & $\begin{array}{l}\text { Kenya } \\
\text { Tanzania } \\
\text { Ghana } \\
\text { Zimbabwe }\end{array}$ & lemon bush & $\begin{array}{l}\text { myrcene } \\
\text { linalool } \\
\alpha \text {-pinene } \\
\text { eucalyptol }\end{array}$ & & & & \\
\hline \multirow[t]{2}{*}{ L. javanica } & & & $\begin{array}{l}\text { alloparinol } \\
\text { camphor }\end{array}$ & $\begin{array}{l}5 \mathrm{mg} / \mathrm{cm} 2 \text { plant extract } \\
\text { applied topically }\end{array}$ & $\begin{array}{l}100 \% \text { protection against Ae. } \\
\text { aegypti for } 8 \text { hours }\end{array}$ & $\begin{array}{l}\text { laboratory } \\
\text { study }\end{array}$ & [117] \\
\hline & & & $\begin{array}{l}\text { limonene } \\
\alpha \text {-terpeneol } \\
\text { verbenone }\end{array}$ & $\begin{array}{l}\text { alcohol plant extract } \\
\text { applied topically }\end{array}$ & $\begin{array}{l}76.7 \% \text { protection against An } \\
\text { arabiensis for } 4 \text { hours }\end{array}$ & $\begin{array}{l}\text { laboratory } \\
\text { study }\end{array}$ & [118] \\
\hline
\end{tabular}


Table 1 An overview of repellent plant efficacy from literature review (Continued)

\begin{tabular}{|c|c|c|c|c|c|c|c|}
\hline \multirow[t]{4}{*}{ L. uckambensis } & & \multirow[t]{4}{*}{ fever tea } & & potted plant & $\begin{array}{l}33.3 \% \text { protection against } A n \text {. } \\
\text { gambiae s.s }\end{array}$ & $\begin{array}{l}\text { semi-field } \\
\text { study in } \\
\text { Kenya }\end{array}$ & [119] \\
\hline & & & & $\begin{array}{l}\text { periodic thermal } \\
\text { expulsion (leaves) }\end{array}$ & $\begin{array}{l}45.9 \% \text { protection against } A n \text {. } \\
\text { gambiae s.s. }\end{array}$ & $\begin{array}{l}\text { semi-field } \\
\text { system in } \\
\text { Kenya }\end{array}$ & {$[62]$} \\
\hline & & & & $\begin{array}{l}\text { periodic direct burning } \\
\text { (leaves) }\end{array}$ & $\begin{array}{l}33.4 \% \text { protection against } A n \text {. } \\
\text { gambiae s.s }\end{array}$ & $\begin{array}{l}\text { semi-field } \\
\text { system in } \\
\text { Kenya }\end{array}$ & {$[62]$} \\
\hline & & & & potted plant & $\begin{array}{l}25.01 \% \text { protection against } A n . \\
\text { gambiae s.l }\end{array}$ & $\begin{array}{l}\text { field study } \\
\text { in Kenya }\end{array}$ & [115] \\
\hline L. cheraliera & & & $\begin{array}{l}\text { eucalyptol } \\
\text { caryophyllene } \\
\text { ipsdienone } \\
\text { p-cymene }\end{array}$ & & & & \\
\hline \multirow[t]{4}{*}{ Lantana camara } & \multirow[t]{4}{*}{$\begin{array}{l}\text { Kenya } \\
\text { Tanzania }\end{array}$} & \multirow{4}{*}{$\begin{array}{l}\text { lantana } \\
\text { spanish flag } \\
\text { West Indian } \\
\text { lantana } \\
\text { Wild sage }\end{array}$} & \multirow[t]{4}{*}{ caryophylene } & potted plant & $\begin{array}{l}32.4 \% \text { protection against } A n \text {. } \\
\text { gambiae s.s }\end{array}$ & $\begin{array}{l}\text { semi-field } \\
\text { study in } \\
\text { Kenya }\end{array}$ & [119] \\
\hline & & & & potted plant & $\begin{array}{l}27.22 \% \text { protection against } A n \text {. } \\
\text { gambiae s.l. }\end{array}$ & $\begin{array}{l}\text { field study } \\
\text { in Kenya }\end{array}$ & [115] \\
\hline & & & & $\begin{array}{l}\text { flower extract in } \\
\text { coconut oil }\end{array}$ & $\begin{array}{l}94.5 \% \text { protection against Ae. } \\
\text { aegypti and Ae. albopictus for } \\
\text { one hour }\end{array}$ & $\begin{array}{l}\text { laboratory } \\
\text { study }\end{array}$ & [120] \\
\hline & & & & $\begin{array}{l}\text { periodic thermal } \\
\text { expulsion (leaves) }\end{array}$ & $\begin{array}{l}42.4 \% \text { protection against } A n \text {. } \\
\text { gambiae s.s }\end{array}$ & $\begin{array}{l}\text { semi-field } \\
\text { study in } \\
\text { Kenya }\end{array}$ & {$[62]$} \\
\hline \multicolumn{8}{|l|}{ LAMIACEAE } \\
\hline \multirow[t]{6}{*}{$\begin{array}{l}\text { Ocimum spp. } O \text {. } \\
\text { americanum }\end{array}$} & \multirow{10}{*}{$\begin{array}{l}\text { Kenya } \\
\text { Tanzania } \\
\text { Zimbabwe } \\
\text { Nigeria } \\
\text { Ghana } \\
\text { Cameroon } \\
\text { Eritrea } \\
\text { Ethiopia (...) }\end{array}$} & \multirow{6}{*}{$\begin{array}{l}\text { Tree basil } \\
\text { nchu avum } \\
\text { lime basil } \\
\text { kivumbasi } \\
\text { Myeni } \\
\text { madongo } \\
\text { African blue } \\
\text { basil } \\
\text { hairy basil }\end{array}$} & \multirow{10}{*}{$\begin{array}{l}\text { p-cymene } \\
\text { estragosl } \\
\text { linalool } \\
\text { linoleic acid } \\
\text { eucalyptol } \\
\text { eugenol } \\
\text { camphor } \\
\text { citral } \\
\text { thujone } \\
\text { limonene } \\
\text { ocimene } \\
\text { and others }\end{array}$} & potted plant & $\begin{array}{l}39.70 \% \text { protection against } A n . \\
\text { gambiae s.s }\end{array}$ & $\begin{array}{l}\text { semi-field } \\
\text { study in } \\
\text { Kenya }\end{array}$ & [119] \\
\hline & & & & potted plant & $\begin{array}{l}37.91 \% \text { protection against } A n . \\
\text { gambiae s.l. }\end{array}$ & $\begin{array}{l}\text { field study } \\
\text { in Kenya }\end{array}$ & [115] \\
\hline & & & & $\begin{array}{l}\text { fresh plants combined } \\
\text { with O. suave bruised } \\
\text { and applied topically }\end{array}$ & $\begin{array}{l}50 \% \text { protection against } A n . \\
\text { gambiae s.l. }\end{array}$ & $\begin{array}{l}\text { field study } \\
\text { in Tanzania }\end{array}$ & [121] \\
\hline & & & & $\begin{array}{l}\text { periodic thermal } \\
\text { expulsion (leaves and } \\
\text { seeds) }\end{array}$ & $\begin{array}{l}43.1 . \% \text { protection against } A n \\
\text { gambiae s.s }\end{array}$ & $\begin{array}{l}\text { semi-field } \\
\text { study in } \\
\text { Kenya }\end{array}$ & {$[62]$} \\
\hline & & & & $\begin{array}{l}\text { periodic direct burning } \\
\text { (leaves and seeds) }\end{array}$ & $\begin{array}{l}20.9 \% \text { protection against } A n \text {. } \\
\text { gambiae s.s }\end{array}$ & $\begin{array}{l}\text { semi-field } \\
\text { study in } \\
\text { Kenya }\end{array}$ & {$[62]$} \\
\hline & & & & $\begin{array}{l}100 \% \text { essential oil } \\
\text { combined with vanillin } \\
5 \% \text { applied topically }\end{array}$ & $\begin{array}{l}100 \% \text { protection against } A e . \\
\text { aegypti for } 6.5 \text { hours } 1 \\
100 \% \text { protection against } C \text {. } \\
\text { quinquefasciatus for } 8 \text { hours } \\
100 \% \text { protection against } A n \text {. } \\
\text { dirus for } 8 \text { hours }\end{array}$ & $\begin{array}{l}\text { laboratory } \\
\text { study }\end{array}$ & {$[34]$} \\
\hline \multirow[t]{4}{*}{ O. suave } & & & & $\begin{array}{l}\text { thermal expulsion } \\
\text { (leaves) }\end{array}$ & $\begin{array}{l}73.6 \% \text { protection from } A n . \\
\text { arabiensis } \\
75.1 \% \text { protection from } A n . \\
\text { pharaoensis }\end{array}$ & $\begin{array}{l}\text { field study } \\
\text { in Ethiopia }\end{array}$ & [114] \\
\hline & & & & direct burning (leaves) & $\begin{array}{l}71.5 \% \text { protection from } A n . \\
\text { arabiensis } \\
79.7 \% \text { protection from An. } \\
\text { pharaoensis }\end{array}$ & $\begin{array}{l}\text { field study } \\
\text { in Ethiopia }\end{array}$ & [114] \\
\hline & & & & $\begin{array}{l}\text { periodic thermal } \\
\text { expulsion (leaves and } \\
\text { seeds) }\end{array}$ & $\begin{array}{l}53.1 \% \text { protection from } A n . \\
\text { gambiae s.s. }\end{array}$ & $\begin{array}{l}\text { semi-field } \\
\text { study in } \\
\text { Kenya }\end{array}$ & {$[62]$} \\
\hline & & & & $\begin{array}{l}\text { periodic direct burning } \\
\text { (leaves and seeds) }\end{array}$ & $\begin{array}{l}28.0 \% \text { protection from } A n . \\
\text { gambiae s.s. }\end{array}$ & $\begin{array}{l}\text { semi-field } \\
\text { study in } \\
\text { Kenya }\end{array}$ & {$[62]$} \\
\hline
\end{tabular}


Table 1 An overview of repellent plant efficacy from literature review (Continued)

\begin{tabular}{|c|c|c|c|c|c|c|c|}
\hline \multirow[t]{3}{*}{ O. basilicum } & & & & $\begin{array}{l}\text { thermal expulsion } \\
\text { (leaves) }\end{array}$ & $\begin{array}{l}78.7 \% \text { protection from } A n . \\
\text { arabiensis } \\
79.2 \% \text { protection from } A n . \\
\text { pharaoensis }\end{array}$ & $\begin{array}{l}\text { field study } \\
\text { in Ethiopia }\end{array}$ & {$[114]$} \\
\hline & & & & direct burning (leaves) & $\begin{array}{l}73.1 \% \text { protection from } A n . \\
\text { arabiensis } \\
70.0 \% \text { protection from } A n . \\
\text { pharaoensis }\end{array}$ & $\begin{array}{l}\text { field study } \\
\text { in Ethiopia }\end{array}$ & [114] \\
\hline & & & & $\begin{array}{l}100 \% \text { essential oil } \\
\text { applied topically }\end{array}$ & $\begin{array}{l}100 \% \text { protection for } 70 \\
\text { minutes }\end{array}$ & $\begin{array}{l}\text { laboratory } \\
\text { study }\end{array}$ & {$[31]$} \\
\hline \multirow[t]{4}{*}{ O. kilimandscharikum } & & & & $\begin{array}{l}\text { thermal expulsion } \\
\text { (leaves and seeds) }\end{array}$ & $\begin{array}{l}44.54 \% \text { protection against } A n \text {. } \\
\text { gambiae s.l. }\end{array}$ & $\begin{array}{l}\text { field study } \\
\text { in Kenya }\end{array}$ & [115] \\
\hline & & & & $\begin{array}{l}\text { thermal expulsion } \\
\text { (leaves and seeds) }\end{array}$ & $\begin{array}{l}37.63 \% \text { protection against } A n . \\
\text { funestus }\end{array}$ & $\begin{array}{l}\text { field study } \\
\text { in Kenya }\end{array}$ & [115] \\
\hline & & & & $\begin{array}{l}\text { periodic thermal } \\
\text { expulsion (leaves and } \\
\text { seeds) }\end{array}$ & $\begin{array}{l}52.0 \% \text { protection against An. } \\
\text { gambiae s.s. }\end{array}$ & $\begin{array}{l}\text { semi-field } \\
\text { study in } \\
\text { Kenya }\end{array}$ & {$[62]$} \\
\hline & & & & $\begin{array}{l}\text { periodic direct burning } \\
\text { (leaves and seeds) }\end{array}$ & $\begin{array}{l}26.4 \% \text { protection against } A n \text {. } \\
\text { gambiae s.s }\end{array}$ & $\begin{array}{l}\text { semi-field } \\
\text { study in } \\
\text { Kenya }\end{array}$ & {$[62]$} \\
\hline O. forskolei & & & & $\begin{array}{l}\text { fresh plants hung } \\
\text { indoors }\end{array}$ & $\begin{array}{l}53 \% \text { protection against } \\
\text { mosquitoes entering human } \\
\text { dwelling }\end{array}$ & $\begin{array}{l}\text { field study } \\
\text { in Eritrea }\end{array}$ & [122] \\
\hline \multirow[t]{3}{*}{$\begin{array}{l}\text { Hyptis spp. Hyptis } \\
\text { suaveolens }\end{array}$} & \multirow{3}{*}{$\begin{array}{l}\text { Kenya } \\
\text { Tanzania } \\
\text { Ghana } \\
\text { The Gambiae }\end{array}$} & \multirow{3}{*}{$\begin{array}{l}\text { bushmint } \\
\text { wild hops } \\
\text { wild } \\
\text { spikenard } \\
\text { hangazimu } \\
\text { hortelã-do- } \\
\text { campo }\end{array}$} & \multirow[t]{3}{*}{ myrcene } & $\begin{array}{l}\text { smouldering on } \\
\text { charcoal }\end{array}$ & $\begin{array}{l}85.4 \% \text { repellency against } \\
\text { mosquitoes for } 2 \text { hours }\end{array}$ & $\begin{array}{l}\text { field study } \\
\text { in Guinea } \\
\text { Bissau }\end{array}$ & [116] \\
\hline & & & & fresh leaves & $\begin{array}{l}\text { 73.2\% repellency against } \\
\text { mosquitoes for } 2 \text { hours }\end{array}$ & $\begin{array}{l}\text { field study } \\
\text { in Guinea } \\
\text { Bissau }\end{array}$ & [116] \\
\hline & & & & $\begin{array}{l}\text { periodic direct burning } \\
\text { (leaves and flowers) }\end{array}$ & $\begin{array}{l}20.8 \% \text { repellency against } A n \text {. } \\
\text { gambiae s.s }\end{array}$ & $\begin{array}{l}\text { semi-field } \\
\text { study in } \\
\text { Kenya }\end{array}$ & {$[62]$} \\
\hline $\begin{array}{l}\text { Mentha spp. M. } \\
\text { piperata }\end{array}$ & $\begin{array}{l}\text { Brazil } \\
\text { Bolivia }\end{array}$ & $\begin{array}{l}\text { hortelã-do- } \\
\text { campo } \\
\text { peppermint }\end{array}$ & & $\begin{array}{l}100 \% \text { essential oil } \\
\text { applied topically }\end{array}$ & $\begin{array}{l}100 \% \text { protection against } A e . \\
\text { aegypti for } 45 \text { minutes }\end{array}$ & $\begin{array}{l}\text { laboratory } \\
\text { study }\end{array}$ & {$[65]$} \\
\hline M. arvensis & & $\begin{array}{l}\text { menta } \\
\text { Japanese } \\
\text { mint }\end{array}$ & & $\begin{array}{l}100 \% \text { essential oil } \\
\text { volatilized in a } \\
\text { kerosene lamp }\end{array}$ & $\begin{array}{l}41 \% \text { protection indoors } \\
\text { against Mansonia spp }\end{array}$ & $\begin{array}{l}\text { field study } \\
\text { in Bolivia }\end{array}$ & {$[17]$} \\
\hline \multirow[t]{7}{*}{$\begin{array}{l}\text { Thymus spp. Th. } \\
\text { vulgaris }\end{array}$} & \multirow{7}{*}{$\begin{array}{l}\text { China } \\
\text { Former Soviet } \\
\text { Union } \\
\text { Korea } \\
\text { Middle-East } \\
\text { Mediterranean }\end{array}$} & \multirow[t]{7}{*}{ thyme } & \multirow{7}{*}{$\begin{array}{l}\alpha \text {-terpinene } \\
\text { carvacrol } \\
\text { thymol } \\
p \text {-cymene } \\
\text { linalool } \\
\text { geraniol }\end{array}$} & $\alpha$-terpinene topically & $\begin{array}{l}97.3 \% \text { protection against Culex } \\
\text { pipiens sallens for } 82 \mathrm{~min}\end{array}$ & \multirow[t]{2}{*}{$\begin{array}{l}\text { laboratory } \\
\text { study }\end{array}$} & \multirow[t]{2}{*}{ [123] } \\
\hline & & & & carvacrol topically & $\begin{array}{l}94.7 \% \text { protection against } C \text {. } \\
\text { pipiens sallens for } 80 \mathrm{~min}\end{array}$ & & \\
\hline & & & & thymol topically & $\begin{array}{l}91.8 \% \text { protection against } C \text {. } \\
\text { pipiens sallens for } 70 \mathrm{~min}\end{array}$ & \multirow[t]{3}{*}{$\begin{array}{l}\text { laboratory } \\
\text { study }\end{array}$} & \multirow[t]{3}{*}{ [123] } \\
\hline & & & & linalool topically & $\begin{array}{l}91.7 \% \text { protection agains } C . \\
\text { pipiens sallens for } 65 \mathrm{~min}\end{array}$ & & \\
\hline & & & & p-cymene & $\begin{array}{l}89.0 \% \text { protection agains } C \text {. } \\
\text { pipiens sallens for } 45.2 \mathrm{~min}\end{array}$ & & \\
\hline & & & & $\begin{array}{l}100 \% \text { essential oil } \\
\text { applied topically }\end{array}$ & $\begin{array}{l}\text { 100\% protection against An. } \\
\text { albimanus for } 105 \text { minutes } \\
\text { and Ae. aegypti for } 135 \\
\text { minutes }\end{array}$ & $\begin{array}{l}\text { laboratory } \\
\text { study }\end{array}$ & {$[65]$} \\
\hline & & & & direct burning (leaves) & $\begin{array}{l}85-09 \% \text { protection for } 60-90 \\
\min \end{array}$ & field study & {$[20]$} \\
\hline Pogostemon spp. & China & Patchouli & & $\begin{array}{l}\text { 100\% essential oil } \\
\text { applied }\end{array}$ & $\begin{array}{l}100 \% \text { protection against } \mathrm{Ae} \text {. } \\
\text { aegypti for } 120 \mathrm{~min}\end{array}$ & $\begin{array}{l}\text { laboratory } \\
\text { study }\end{array}$ & {$[31]$} \\
\hline Pogostemon cablin & $\begin{array}{l}\text { India } \\
\text { Malaysia } \\
\text { Thailand }\end{array}$ & Oriza & & topically & $\begin{array}{l}\text { 100\% protection against } C \text {. } \\
\text { quinquefasciatus for } 150 \text { min } \\
100 \% \text { protection against } A n . \\
\text { dirus for } 710 \text { minutes }\end{array}$ & & \\
\hline
\end{tabular}


Table 1 An overview of repellent plant efficacy from literature review (Continued)

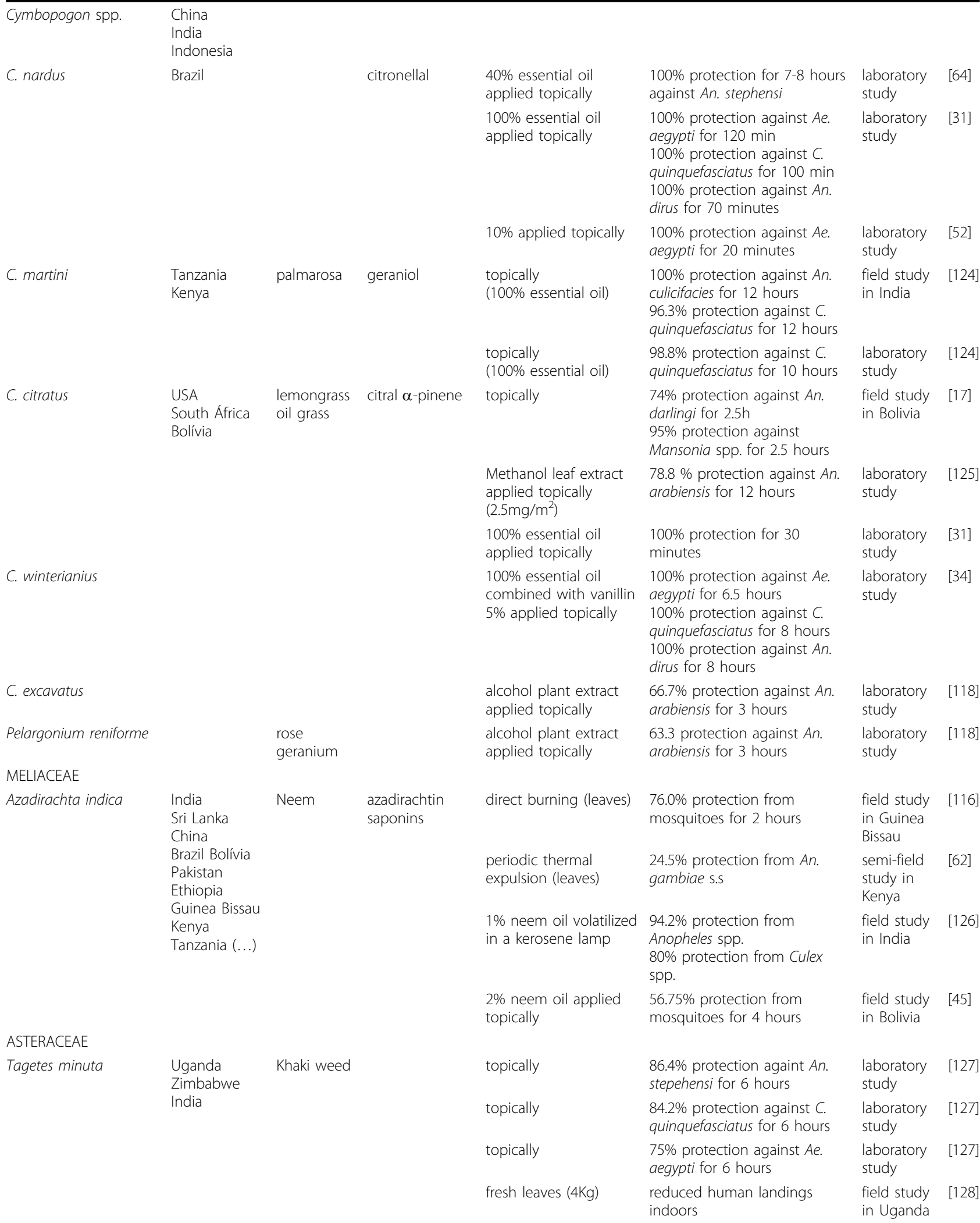


Table 1 An overview of repellent plant efficacy from literature review (Continued)

\begin{tabular}{|c|c|c|c|c|c|c|c|}
\hline $\begin{array}{l}\text { Artemisia spp. } \\
\text { A. vulgaris }\end{array}$ & $\begin{array}{l}\text { India } \\
\text { Egypt } \\
\text { Italy } \\
\text { Canada } \\
\text { USA }\end{array}$ & $\begin{array}{l}\text { mugwort } \\
\text { wormwood } \\
\text { St. Johns } \\
\text { plant } \\
\text { Old uncle } \\
\text { henry } \\
\text { Sailors } \\
\text { tobacco }\end{array}$ & $\begin{array}{l}\text { camphor } \\
\text { linalool } \\
\text { terpenen-4-ol } \\
\alpha \text {-and } \beta \text { - } \\
\text { thujone } \\
\beta \text {-pinene }\end{array}$ & & & & \\
\hline A. monosperma & Siberia Brazil & $\begin{array}{l}\text { Felon herb } \\
\text { Naughty } \\
\text { man }\end{array}$ & $\begin{array}{l}\text { myrcene } \\
\text { limonene } \\
\text { cineol }\end{array}$ & $\begin{array}{l}5 \% \text { leave extract } \\
\text { applied topically }\end{array}$ & $100 \%$ protection for 4 hours & $\begin{array}{l}\text { field study } \\
\text { in Egypt }\end{array}$ & 112 \\
\hline \multicolumn{8}{|l|}{ CAESALPINIACEAE } \\
\hline \multirow[t]{2}{*}{ Daniellia oliveri } & $\begin{array}{l}\text { Guinea-Bissau } \\
\text { The Gambiae }\end{array}$ & $\begin{array}{l}\text { churai } \\
\text { santão } \\
\text { santang }\end{array}$ & & direct burning (bark) & $\begin{array}{l}77.9 \% \text { protection against } \\
\text { mosquitoes for } 2 \text { hours }\end{array}$ & $\begin{array}{l}\text { field study } \\
\text { in Guinea } \\
\text { Bissau }\end{array}$ & [116] \\
\hline & & santango & & direct burning (bark) & $\begin{array}{l}77 \% \text { protection against } \\
\text { mosquitoes }\end{array}$ & $\begin{array}{l}\text { field study } \\
\text { in The } \\
\text { Gambiae }\end{array}$ & [130] \\
\hline $\begin{array}{l}\text { FABACEAE } \\
\text { Glycine max }\end{array}$ & Worldwide & Soya & & $2 \%$ soya bean oil & $\begin{array}{l}\text { 100\% protection against } A e . \\
\text { aegypti for } 95 \text { minutes }\end{array}$ & $\begin{array}{l}\text { laboratoty } \\
\text { study }\end{array}$ & {$[52]$} \\
\hline \multirow[t]{2}{*}{$\begin{array}{l}\text { RUTACEAE } \\
\text { Zanthoxylum limonella }\end{array}$} & Thailand & makaen & & $\begin{array}{l}\text { 100\% essential oil } \\
\text { applied topically }\end{array}$ & $\begin{array}{l}\text { 100\% protection against } \mathrm{Ae} \text {. } \\
\text { aegypti for } 120 \text { min } \\
100 \% \text { protection against } \mathrm{C} . \\
\text { quinquefasciatus for } 170 \mathrm{~min}\end{array}$ & $\begin{array}{l}\text { laboratory } \\
\text { study }\end{array}$ & {$[31]$} \\
\hline & & & & $\begin{array}{l}10 \% \text { essential oil } \\
\text { combined with } 10 \% \\
\text { clove oil }\end{array}$ & $\begin{array}{l}100 \% \text { protection against An. } \\
\text { dirus for } 190 \text { minutes }\end{array}$ & $\begin{array}{l}\text { laboratory } \\
\text { study }\end{array}$ & {$[64]$} \\
\hline Citrus hystrix & $\begin{array}{l}\text { Indonesia } \\
\text { Malaysia } \\
\text { Thailand } \\
\text { Laos }\end{array}$ & $\begin{array}{l}\text { Kaffir lime } \\
\text { Limau } \\
\text { purut }\end{array}$ & & $\begin{array}{l}100 \% \text { essential oil } \\
\text { combined with vanillin } \\
5 \% \text { applied topically }\end{array}$ & $\begin{array}{l}\text { 100\% protection against } A n . \\
\text { stephensi for } 8 \text { hours } \\
100 \% \text { protection against Ae. } \\
\text { aegypti for } 3 \text { hours } \\
100 \% \text { protection against C. } \\
\text { quinquefasciatus for } 1.5 \text { hours } \\
100 \% \text { protection against } A n . \\
\text { dirus for } 2.5 \text { hours }\end{array}$ & $\begin{array}{l}\text { laboratory } \\
\text { study }\end{array}$ & {$[34]$} \\
\hline $\begin{array}{l}\text { ZINGIBERACEAE } \\
\text { Curcuma longa }\end{array}$ & & $\begin{array}{l}\text { Turmeric } \\
\text { Curcuma } \\
\text { Indian } \\
\text { saffron }\end{array}$ & & $\begin{array}{l}100 \% \text { essential oil } \\
\text { combined with vanillin } \\
5 \% \text { applied topically }\end{array}$ & $\begin{array}{l}100 \% \text { protection against } A e . \\
\text { aegypti for } 4.5 \text { hours } \\
100 \% \text { protection against } C . \\
\text { quinquefasciatus for } 8 \text { hours } \\
100 \% \text { protection against } A n \text {. } \\
\text { dirus for } 8 \text { hours }\end{array}$ & $\begin{array}{l}\text { laboratory } \\
\text { study }\end{array}$ & {$[34]$} \\
\hline
\end{tabular}

travellers to disease endemic areas should not be recommended citronella-based repellents [32]. In contrast, for those communities where more efficacious alternatives are not available, or are prohibitively expensive, the use of citronella to prevent mosquito bites may provide important protection from disease vectors [17].

The second way to use volatile plant repellents is to continuously evaporate them. Citronella and geraniol candles are widely sold as outdoor repellents, however field studies against mixed populations of nuisance mosquitoes show reductions in biting around 50\%, although they do not provide significant protection against mosquito bites [37-39].

\section{Neem}

Neem is widely advertised as a natural alternative to DEET [40], and it has been tested for repellency against range of arthropods of medical importance, with variable results (Table 1). Several field studies from India have shown very high efficacy of Neem-based preparations [41-43], contrasting with findings of intermediate repellency by other researchers $[44,45]$. However, these contrasting results may be due to differing methodologies, and the solvents used to carry the repellents. The EPA has not approved Neem for use as a topical insect repellent. It has a low dermal toxicity, but can cause skin irritation, such as dermatitis when used undiluted [46]. Due to the paucity of reliable studies, Neem oil is not recommended as an effective repellent for use by travellers to disease endemic areas [32], although it may confer some protection against nuisance biting mosquitoes.

\section{Natural oils and emulsions}

Several oils have shown repellency against mosquitoes. It is likely that they work in several ways 1 ) by reducing short range attractive cues i.e. kairomones, water vapour 
and temperature [47-49]; 2) by reducing the evaporation and absorption of repellent actives due to the presence of long-chained fatty molecules [50]; 3) by containing fatty acids are known to be repellent to mosquitoes at high concentrations [51]. Bite Blocker, a commercial preparation containing glycerin, lecithin, vanillin, oils of coconut, geranium, and $2 \%$ soybean oil can achieve similar repellency to DEET, providing 7.2 hours mean protection time against a dengue vector and nuisance biting mosquitoes in one study [44], and protection for 1.5 hours, equivalent to that of low concentration DEET in a second study [52]. It would appear that the soybean oil in Bite Blocker helps only contributes to repellency as it is not repellent when evaluated on its own [53]. Soybean oil is not EPA registered, but it has low dermal toxicity, although no recommended maximum exposure or chronic exposure limits have been established [54]. Other plant-based oils that have shown some repellent efficacy are coconut oil, palm nut oils [55] and andiroba oil [56], although all of these three oils are far less effective than DEET, they may be useful as carriers for other repellent actives as they are cheap and contain unsaturated fatty acids and emulsifiers that improve repellent coverage and slow evaporation of volatile repellent molecules [50,53,57].

\section{Essential oils}

Essential oils distilled from members of the Lamiaceae (mint family that includes most culinary herbs), Poaceae (aromatic grasses) and Pinaceae (pine and cedar family) are commonly used as insect repellents throughout the globe (Table 1). Many members of these families are used in rural communities through burning or hanging them within homes [58-62]. In Europe and North America there is a strong history of use of the oils dating back to Ancient times. Almost all of the plants used as repellents are also used for food flavouring or in the perfume industry, which may explain the association with these oils as safer natural alternatives to DEET despite many oils causing contact dermatitis (Table 2 [63]). Many commercial repellents contain a number of plant essential oils either for fragrance or as repellents including peppermint, lemongrass, geraniol, pine oil, pennyroyal, cedar oil, thyme oil and patchouli. The most effective of these include thyme oil, geraniol, peppermint oil, cedar oil, patchouli and clove that have been found to repel malaria, filarial and yellow fever vectors for a period of 60-180 mins [64-66]. Most of these essential oils are highly volatile and this contributes to their poor longevity as mosquito repellents. However, this problem can be addressed by using fixatives or careful formulation to improve their longevity. For example, oils from turmeric and hairy basil with addition of $5 \%$ vanillin repelled 3 species of mosquitoes under cage conditions for a period of 6-8 hours depending on the mosquito species [34]. Although essential oils are exempt from registration through the EPA, they can be irritating to the skin and their repellent effect is variable, dependent on formulation and concentration. Repellents containing only essential oils in the absence of an active ingredient such as DEET should not be recommended as repellents for use in disease endemic areas, and those containing high levels of essential oils could cause skin irritation, especially in the presence of sunlight.

\section{Considerations for repellent testing methodology}

In a Pubmed search using the terms "plant" and "repellent" and "mosquito" in the past 5 years, 87 results were shown. These studies can be broken down into a series of categories: 1) standard ethnobotanical studies and evaluations of plants that are traditionally used to repel mosquitoes [17,67-70]; 2) standard dose response [33] laboratory evaluations of solvent extractions of plants without DEET positive controls [71]; 3) standard dose response [33] laboratory evaluations of solvent or extractions or essential oils of plants with DEET positive controls [72] coupled with GC-MS (coupled gas chromatography-mass spectrometry) [73]; 4) laboratory evaluations using time to first bite method [74] comparing the plant repellents to DEET [75] and in addition several of those studies also analysed the constituents of the oil through GC-MS $[76,77]$. In addition there were a large number of studies that did not use the accepted standard methodology [78] (Table 3), and should be interpreted with caution. Only two studies considered safety [79] or adverse effects [80] and only one study considered randomization and blinding [52], and almost all repellent studies did not consider the number of human participants needed to minimize sampling error [81]. It is important for the future development of plant based repellents that the standard WHO methodology is followed [78], including a DEET control to allow simple comparison of multiple studies, and reporting of standard errors to understand the reliability of that repellent compound to provide the observed protection.

\section{Some fallacies about plant based or natural repellents}

It is commonly assumed that plant-based repellents are safer than DEET because they are natural. However, some natural repellents are safer than others, and it cannot be assumed that natural equates to safe [18]. DEET has undergone stringent testing and has a good safety profile. An estimated 15 million people in the U.K., 78 million people in the U.S.A. [82], and 200 million people globally use DEET each year [83]. Provided that DEET is used safely, i.e. it is applied to the skin at the correct dose (such 
Table 2 Some common ingredients in natural repellents that may be hazardous. Reproduced with permission from [63]

\begin{tabular}{|c|c|c|c|}
\hline Common Name & Scientific Name & $\begin{array}{l}\text { Safe } \\
\text { Concentration }\end{array}$ & Hazard \\
\hline Anise & Pimpinella anisum & $3.6 \%$ & Based on $0.11 \%$ methyl eugenol; carcinogen \\
\hline Basil & Ocimum sp & $0.07 \%$ & Based on 6\% methyl eugenol; carcinogen \\
\hline Bergamot & Citrus aurantium bergamia & $0.4 \%$ & Sensitising and phototoxic; skin irritant \\
\hline Cajeput & Melaleuca alternifolia & $0.004 \%$ & Based on 97\% methyl eugenol; carcinogen \\
\hline Cedar & Chamaecyparis nootkatensis & $1 \%$ & Likely allergenic contaminants if nootkatone not $98 \%$ pure \\
\hline Cassia & Cinnamonium cassia & $0.2 \%$ or $9 \%$ & Sensitising skin irritant \\
\hline Citronella & Cymbopogon nardus & $2 \%$ & $\begin{array}{l}\text { Safety is controversial; based on } 0.2 \% \text { methyl eugenol or } 1.3 \% \text { citral; } \\
\text { sensitising skin irritant }\end{array}$ \\
\hline Citronella (Java) & Cymbopogon winterianius & $2 \%$ & Based on $0.2 \%$ methyl eugenol; carcinogen \\
\hline Citrus oils & Citrus sp & $16-25 \%$ & Based on $0.005 \%-0.0025 \%$ bergapten; phototoxic skin irritant \\
\hline Clove & Syzyguim aromaticum & $0.5 \%$ & Based on 92\% eugenol; sensitising skin irritant \\
\hline Fever tea, lemon bush & Lippia javanica & $2 \%$ & Based on $5 \%$ citral in related species; sensitising skin irritant \\
\hline Geranium & Pelargonium graveolens & $6 \%$ & Based on $1.5 \%$ citral; sensitising skin irritant \\
\hline Ginger & Zingiber sp & $12 \%$ & Based on $0.8 \%$ citral; sensitising skin irritant \\
\hline Huon oil, Macquarie pine & Langarostrobus franklini & $0.004 \%$ & Based on $98 \%$ methyl eugenol; carcinogen \\
\hline Lemongrass & Cymbopogon citratus & $0.1 \%$ & Based on $90 \%$ citral; sensitising skin irritant \\
\hline Lime & Citrus aurantifolia & $0.7 \%$ & Phototoxic skin irritant \\
\hline Litsea & Litsea cubeba & $0.1 \%$ & Based on 78\% citral; sensitising skin irritant \\
\hline Marigold & Tagates minuta & $0.01 \%$ & Phototoxic skin irritant \\
\hline $\begin{array}{l}\text { Mexican tea, American } \\
\text { wormseed }\end{array}$ & Chenopodium ambrosioides & Prohibited & Toxic \\
\hline Mint & Mentha piperata and spicata & $2 \%$ & Based on 0.1\% trans-2-hexenal; sensitising skin irritant \\
\hline Nutmeg & Myristica fragrans & $0.4 \%$ & Based on 1\% methyl eugenol; carcinogen \\
\hline Palmarosa & Cymbopogon martini & $16 \%$ & Based on 1.2\% farnesol; sensitizing skin irritant \\
\hline Pennyroyal & $\begin{array}{l}\text { Mentha pulegium or Hedeoma } \\
\text { pulegioides }\end{array}$ & Prohibited & Toxic \\
\hline Pine & Pinus sylvestris & $\begin{array}{l}\text { Prepare with } \\
\text { antioxidants }\end{array}$ & Oxidation creates phototoxic skin irritants \\
\hline Rosemary & Rosemarinus officinalis & $36 \%$ & Based on $0.011 \%$ methyl eugenol; carcinogen \\
\hline Rue & Ruta chalepensis & $0.15 \%$ & Based on presence of psoralenes; phototoxic skin irritant \\
\hline Thyme & Thymus vulgaris & $2 \%$ & Based on 0.1\% trans-2-hexenal; sensitising skin irritant \\
\hline Violet & Viola odorata & $2 \%$ & Based on 0.1\% trans-2-hexenal; sensitising skin irritant \\
\hline Ylang-ylang & Canagium odoratum & $2 \%$ & Based on $4 \%$ farnesol; sensitizing skin irritant \\
\hline
\end{tabular}

as that in a commercial preparation) and it is not swallowed or rubbed into the mucous membranes then it does not cause adverse effects [84]. DEET has been used since 1946 with a tiny number of reported adverse effects, many of which had a history of excessive or inappropriate use of repellent $[85,86]$. Its toxicology has been more closely scrutinized than any other repellent, and it has been deemed safe for human use [82,87], including use on children [88], pregnant women [89], and lactating women [84]. In contrast, plant-based repellents do not have this rigorously tested safety record, with most being deemed safe because they have simply been used for a long time [90]. However, many plant-based repellents contain compounds that should be used with caution (Table 1).

It is also commonly stated that plant based repellents are better for the environment than synthetic molecules.
While plant volatiles are naturally derived, distillation requires biomass energy, extraction commonly uses organic solvents that must be disposed of carefully, growing the plants uses agrichemicals, such as fertilizers and pesticides (unless sourced from a sustainable and organic source). However, if carefully practiced, cash cropping of plants used for repellents provides a vital source of income for small scale farmers in developing countries [91] and can have beneficial environmental impact when planted in intercropping systems to prevent soil erosions [92]. Therefore, it is important to carefully source of repellent plants to avoid pitfalls associated with unsustainable cropping practices. Another common misconception is that garlic is an effective repellent. It does have a moderate repellent effect when rubbed on the skin [93], although there are far more 
Table 3 Guidelines on repellent testing adapted from [78]

\begin{tabular}{l} 
WHOPES approved repellent testing methodology \\
\hline Laboratory Testing \\
Use $20 \%$ deet in ethanol as a positive comparison \\
Human subjects preferable to reflect the end user \\
Before the test the test area of skin should be washed with unscented soap then rinsed with $70 \%$ ethanol / isopropyl alcohol \\
Mosquitoes should be reared under standard $27 \pm 2$ C temperature, $\geq 80 \pm 10 \%$ relative humidity, and a 12:12 (light:dark) photoperiod. \\
Mosquitoes should be 3 to 5 days old, nulliparous females, starved for 12 hours preceding the test \\
Tests should be conducted with three or more species \\
$40 \times 40 \times 40$ cm cages with 50 - 100 mosquitoes for effective dose testing \\
$40 \times 40 \times 40$ cm cages with 200 - 250 mosquitoes for complete protection time testing \\
Control arms should be used to estimate mosquito readiness to feed \\
Treatment arms should be offered to mosquitoes after avidity has been measured \\
Field Testing \\
Use $20 \%$ deet in ethanol as a positive comparison \\
Human subjects preferable to reflect the end user \\
Before the test the test area of skin should be washed with unscented soap then rinsed with $70 \%$ ethanol / isopropyl alcohol \\
Volunteers should sit $>20$ metres apart \\
Design should be completely randomised \\
Trials should be conducted with medium biting pressures of representative vector species \\
All participants should be recruited on informed consent from the local area and be provided with malaria prophylaxis \\
In all testing monitoring of adverse effects should be carried out \\
\hline
\end{tabular}

effective repellents available that also have a more pleasing odour. The consumption of garlic however, has not been shown to be effective at repelling mosquitoes.

\section{Promising developments in plant based repellents}

The field of plant-based repellents is moving forward as consumers demand means of protection from arthropod bites that are safe, pleasant to use and environmentally sustainable. Perhaps the most important consideration is improving the longevity of those repellents that are effective but volatile such as citronella. Several studies looked at improving formulations of plant oils to increase their longevity through development of nanoemulsions [35,94], improved formulations and fixatives [95-97]; while alternate uses such as spatial activity [98-102] and excitorepellency [103,104] have also been investigated. There has been a single clinical study of PMD to lower malaria incidence [26]. This is an exciting discovery since PMD may be recovered from distillation of leaves of E. citroidora or chemical modification of citronellal [105]- available from plants of the genus Cymbopogon. These plants are already commercially cropped in malaria endemic countries including South America, especially Brazil (6 million trees), southern China, India, Sri Lanka, Congo (Zaire), Kenya and most countries in southern Africa, where it is grown for essential oil production and timber [106]. Local production of insect repellent would remove the high cost of importation in developing countries.
New developments have also been seen in understanding the function of plant-based repellents in insects. Several studies have investigated the behavioural mode of action of repellents through structure-activity studies of contact versus spatial repellency [107], or olfactometry that demonstrated that DEET inhibited mosquito response to human odour whereas Ocimum forskolei repels but does not inhibit response to human odour [108]. A further study demonstrates that citronellal directly activates cation channels [10], which is similar to the excitorepellent effect of pyrethrin - another plant based terpine [109], but contrasts with the inhibition effect of DEET [3].

The field of repellent development from plants is extremely fertile due to wealth of insecticidal compounds found in plants as defences against insects [2]. The modern pyrethroids that are the mainstay of the current malaria elimination program that is making excellent progress [110], are synthetic analogues based on the chemical structure of pyrethrins, discovered in the pyrethrum daisy, Tanacetum cinerarifolium from the Dalmation region and Tanacetum coccineum of Persian origin. The insecticidal component comprising six esters (pyrethrins) is found in tiny oil-containing glands on the surface of the seed case in the flower head to protect the seed from insect attack. Pyrethrins are highly effective insecticides, that are relatively harmless to mammals [111], although it must be emphasised that many other plant produce compounds that are highly toxic to mammals and / or irritating to the skin, and 
natural does not equate to safe. In the past few years, a plant derived repellent, PMD has been proven to be suitably efficacious and safe to compete with DEET in the field of disease prevention, and repellents have been recognised by WHO as a useful disease prevention tool to complement insecticide-based means of vector control. The field of plant-based repellent evaluation and development had become far more rigorous in recent years and developments in methods of dispensing plantbased volatiles means that extension in the duration of repellency and consequent efficacy of plant-based repellents will be possible in future.

\section{Acknowledgements and funding}

Authors receive salary support from Bill and Melinda Gates Foundation 51431. We would like to thank Coronel Mustapha Debboun for permission to reproduce Table 2 and the two anonymous reviewers who greatly improved the manuscript through their comments and suggestions. This article has been published as part of Malaria Journal Volume 10 Supplement 1, 2011: Natural products for the control of malaria. The full contents of the supplement are available online at http://www. malariajournal.com/supplements/10/S1

\section{Author details}

'Disease Control Department, London School of Hygiene and Tropical Medicine, Keppel Street, London WC1E 7HT, UK. ${ }^{2}$ Biomedical and Environmental Thematic Group, Ifakara Health Institute, Ifakara, Morogoro, Tanzania.

\section{Author's contributions}

Manuscript drafted by MFM and SJM.

\section{Competing interests}

The authors declare that they have no competing interests

Published: 15 March 2011

\section{References}

1. Pichersky E, Gershenzon J: The formation and function of plant volatiles: perfumes for pollinator attraction and defense. Curr Opinion Plant Biology 2002, 5:237-243.

2. Harrewijn $P$, Minks AK, Mollema C: Evolution of plant volatile production in insect-plant relationships. Chemoecology 1995, 5:55-73.

3. Ditzen M, Pellegrino M, Vosshall LB: Insect odorant receptors are molecular targets of the insect repellent deet. Science 2008 319:1838-1842.

4. Hallem EA, Dahanukar A, Carlson JR: Insect odor and taste receptors. Annu Rev Entomol 2006, 51:113-135.

5. Pitts RJ, Fox AN, Zwiebel L: A highly conserved candidate chemoreceptor expressed in both olfactory and gustatory tissues in the malaria vector, Anopheles gambiae. Proc Natl Acad Sci U S A 2004, 101:5058-5063.

6. Gatehouse JA: Plant resistance towards insect herbivores: a dynamic interaction. New Phytologist 2002, 156.

7. Carey AF, Wang G, Su CY, Zwiebel LJ, Carlson JR: Odorant reception in the malaria mosquito Anopheles gambiae. Nature 2010, 464:66-71.

8. Logan JG, Stanczyk NM, Hassanali A, Kemei J, Santana AEG, Ribeiro KAL, Pickett JA, Mordue (Luntz) JA: Arm-in-cage testing of natural humanderived mosquito repellents. Malar J 2010, 9:239.

9. Syed Z, Leal WS: Mosquitoes smell and avoid the insect repellent DEET. Proc Natl Acad Sci U S A 2008, 10:1073.

10. Kwon Y, Kim SH, Ronderos DS, Lee Y, Akitake B, Woodward OM, Guggino WB, Smith DP, Montell C: Drosophila TRPA1 channel is required to avoid the naturally occurring insect repellent citronellal. Curr Biol 2010, 20:1672-1678.
11. Gershenzon J, Dudareva N: The function of terpene natural products in the natural world. Nature Chemical Biology 2007, 3:408-414.

12. Lee SE, Lee BH, Choi WS, Park BS, Kim JG, Campbell BC: Fumigant toxicity of volatile natural products from Korean spices and medicinal plants towards the rice weevil, Sitophilus oryzae (L). Pest Manag Sci 2001, 57:548-553.

13. Moore SJ, Lenglet A, Hill N: Plant-Based Insect Repellents. In Insect Repellents: Principles Methods, and Use. Boca Raton Florida: CRC Press; Debboun M, Frances SP, Strickman D 2006 .

14. Herodotus: Herodotus. The Histories. Penguin; 1996.

15. Owen T: Geoponika: Agricultural Pursuits. 1805 [http://www.ancientlibrary. com/geoponica/index.html].

16. Johnson T: CRC Ethnobotany Desk Reference. Boca Raton, Florida: CRC Press; 1998.

17. Moore SJ, Hill N, Ruiz C, Cameron MM: Field Evaluation of Traditionally Used Plant-Based Insect Repellents and Fumigants Against the Malaria Vector Anopheles darlingi in Riberalta, Bolivian Amazon. J Med Entomol 2007, 44(4):624-630.

18. Trumble JT: Caveat emptor: safety considerations for natural products used in arthropod control. Am Entomol 2002, 48:7-13.

19. Casas A, Valiente-Banuet A, Viveros JL, Caballero J, Cortes L, Davila P, Lira R, Rodriguez I: Plant resources of the Tehuacan-Cuicatlan Valley, Mexico. Econ Bot 2001, 55:129-166.

20. Curtis CF: Traditional use of repellents. In Appropriate technology in vector control. Boca Raton, Florida: CRC Press; Curtis CF 1990:81-82.

21. Vieira IG: Estudo de caracteres silviculturais e de produção de óleo essencial de progênies de Corymbia citriodora (Hook) K.D.Hill \& L.A.S. Johnson procedente de Anhembi SP - Brasil, Ex. Atherton QLD Austrália. Universidad de Sao Paulo, Escola Superior de Agricultura Luiz de Queiroz; 2004

22. Barasa SS, Ndiege IO, Lwande W, Hassanali A: Repellent activities of stereoisomers of $p$-menthane-3,8-diols against Anopheles gambiae (Diptera: Culicidae). J Med Entomol 2002, 39:736-741.

23. Carroll SP, Loye J: PMD, a registered botanical mosquito repellent with deet-like efficacy. J Am Mosa Control Assoc 2006, 22:507-514.

24. Phasomkusolsil S, Soonwera M: Insect repellent activity of madicinal plant oils against Aedes aegypti (Linn.), Anopheles minimus (Theobald) and Culex quinquefasciatus Say based based on protection time and biting rate. Southeast Asian J Trop Med Public Health 2010, 41:831-840.

25. Emily Zielinski-Gutierrez RAW, Roger S.Nasci: Protection against mosquitoes, ticks and other insects and arthropods. CDC Health Information for International Travel ("The Yellow Book") Atlanta: Centres for Disease Control and Prevention; 2010

26. Hill N, Lenglet A, Arnez AM, Cainero I: Randomised, double-blind control trial of p-menthane diol repellent against malaria in Bolivia. BMJ 2007, 55.

27. EPA: p-Menthane-3,8-diol (011550) Fact Sheet [http://www.epa.gov/ oppbppd1/biopesticides/ingredients/factsheets/factsheet_ 011550.htm].

28. Dictionary.com: website: [http://dictionary.reference.com/browse/ citronella]..

29. Covell G: Anti-mosquito measures with special reference to India. Health Bulletin 1943, 11.

30. EPA: Registration Eligability Descision Document: Oil of Citronella [http://www.epa.gov/oppsrrd1/REDs/factsheets/3105fact.pdf].

31. Trongtokit $Y$, Rongsriyam $Y$, Komalamisra N, Apiwathnasorn C: Comparative repellency of 38 essential oils against mosquito bites. Phytother Res 2005, 19:303-309.

32. Goodyer LI, Croft AM, Frances SP, Hill N, Moore SJ, Onyango SP, Debboun M: Expert review of the evidence base for arthropod bite avoidance. J Travel Med 2010, 17:1708-8305

33. Curtis CF, Lines JD, ljumba J, Callaghan A, Hill N, Karimzad MA: The relative efficacy of repellents against mosquito vectors of disease. Med Vet Entomol 1987, 1:109-119.

34. Tawatsin A, Wratten SD, Scott RR, Thavara U, Techadamrongsin Y: Repellency of volatile oils from plants against three mosquito vectors. J Vector Ecol 2001, 26:76-82.

35. Sakulku U, Nuchuchua O, Uawongyart N, Puttipipatkhachorn S, Soottitantawat A, Ruktanonchai U: Characterization and mosquito repellent activity of citronella oil nanoemulsion. Int J Pharm 2009, 372:105-111. 
36. Miro Specos MM, Garcia JJ, Tornesello J, Marino P, Della Vecchia M, Defain Tesoriero MV, Hermida LG: Microencapsulated citronella oil for mosquito repellent finishing of cotton textiles. Trans $R$ Soc of Trop Med Hyg 2010, 104:653-658

37. Lindsay LR, Surgeoner GA, Heal JD, Gallivan GJ: Evaluation of the efficacy of $3 \%$ citronella candles and $5 \%$ citronella incense for protection against field populations of Aedes mosquitoes. J Am Mosq Control Assoc 1996, 12:293-294.

38. Müller GC, Junnila A, Kravchenko VD, Revay EE, Butler J, Orlova OB, Weiss RW, Schlein Y: Ability of essential oil candles to repel biting insects in high and low biting pressure environments. J Am Mosq Control Assoc 2008, 24:154-160.

39. Jensen T, Lampman R, Slamecka MC, Novak RJ: Field efficacy of commercial antimosquito products in Illinois. J Am Mosq Control Assoc 2000, 16:148-152.

40. Ava T: Neem oil: a safe alternative to Deet [http://trinityava.com/wpcontent/.../Neem-for-Outdoor-Protection-2009.07.pdf]. Book Neem oil: a safe alternative to Deet City; 2009 [http://trinityava.com/wp-content/ .../Neem-for-Outdoor-Protection-2009.07.pdf].

41. Singh N, Mishra AK, Saxena A: Use of neem cream as a mosquito repellent in tribal areas of central India. Indian J Malariol 1996, 33:99-102.

42. Sharma VP, Ansari MA, Razdan RK: Mosquito repellent action of neem (Azadirachta indica) oil. J Am Mosq Control Assoc 1993, 9:359-360.

43. Caraballo AJ: Mosquito repellent action of Neemos. J Am Mosa Control Assoc 2000, 16:45-46.

44. Barnard DR, Xue RD: Laboratory evaluation of mosquito repellents against Aedes albopictus, Culex nigripalpus, and Ochierotatus triseriatus (Diptera: Culicidae). J Med Entomol 2004, 41:726-730.

45. Moore SJ, Lenglet A, Hill N: Field evaluation of three plant-based insect repellents against malaria vectors in Vaca Diez Province, the Bolivian Amazon. J Am Mosa Control Assoc 2002, 18:107-110.

46. Reutemann $P$, Ehrlich A: Neem oil: an herbal therapy for alopecia causes dermatitis. Dermatitis 2008, 19:E12-15.

47. Eiras $A E$, Jepson PC: Responses of female Aedes aegypti (Diptera: Culicidae) to host odours and convection currents using an olfactometer bioassay. Bull Entomol Res 1994, 84:207-211.

48. Davis EE, Bowen MF: Sensory physiological basis for attraction in mosquitoes. J Am Mosq Control Assoc 1994, 10:316-325.

49. Wright $\mathrm{RH}$, Kellogg FE: Response of Aedes aegypti to moist convection currents. Nature 1962, 194:402-403.

50. Dremova VP, Markina W, Kamennov NA: How evaporation and absorption affect the formulation of various insect repellents. Int Pest Cont 1971, 13:13-16.

51. Skinner WA, Tong HC, Maibach HI, Skidmore DL: Human skin surface lipid fatty acids - mosquito repellents. Cell Mol Life Sci 1970, 26:728-730.

52. Fradin MS, Day JF: Comparative efficacy of insect repellents against mosquito bites. $N$ Engl J Med 2002, 347:13-18.

53. Campbell C, Gries G: Is soybean oil an effective repellent against Aedes aegypti? Can Entomol 2010, 142:405-414.

54. Biconet : MSDS Bite Blocker Spray [http://www.biconet.com/personal/ infosheets/biteBlockerSprayMSDS.pdf]. Book MSDS Bite Blocker Spray

55. Konan YL, Sylla MS, Doannio JM, Traoré S: Comparison of the effect of two excipients (karite nut butter and vaseline) on the efficacy of Cocos nucifera, Elaeis guineensis and Carapa procera oil-based repellents formulations against mosquitoes biting in Ivory Coast. Parasite 2003, 10:181-184.

56. Miot HA, Batistella RF, Batista Kde A, Volpato DE, Augusto LS, Madeira NG, Haddad VJ, Miot LD: Comparative study of the topical effectiveness of the Andiroba oil (Carapa guianensis) and DEET 50\% as repellent for Aedes sp. Rev Inst Med Trop Sao Paulo 2004, 46:235-236.

57. Reifenrath WG, Hawkins GS, Kurtz MS: Evaporation and skin penetration characteristics of mosquito repellent formulations. J Am Mosq Control Assoc 1989, 5:45-51.

58. Oparaocha ET, Iwu I, Ahanakuc JE: Preliminary study on mosquito repellent and mosquitocidal activities of Ocimum gratissimum (L.) grown in eastern Nigeria. J Vector Borne Dis 47:45-50.

59. Ntonifor NN, Ngufor CA, Kimbi HK, Oben BO: Traditional use of indigenous mosquito-repellents to protect humans against mosquitoes and other insect bites in a rural community of Cameroon. East Afr Med J 2006, 83:553-558.
60. Lukwa N: Do traditional mosquito repellent plants work as mosquito larvicides. Central African Journal of Medicine 1994, 40:306-309.

61. Marazanye T, Chagwedera TE, Adotey J: Wild local plant derivatives as an alternative to conventional mosquito repellent. Central African Journal of Medicine 1988, 34

62. Seyoum A, Palsson K, Kung'a S, Kabiru EW, Lwande W, Killeen GF, Hassanali A, Knols BG: Traditional use of mosquito-repellent plants in western Kenya and their evaluation in semi-field experimental huts against Anopheles gambiae: ethnobotanical studies and application by thermal expulsion and direct burning. Trans R Soc Trop Med Hyg 2002, 96:225-231.

63. Strickman D, Frances SP, Debboun M: Chapter 8: Put on something natural. Prevention of bugs, bites, stings and disease New York: Oxford University Press; 2009.

64. Trongtokit $Y$, Curtis CF, Rongsriyam Y: Efficacy of repellent products against caged and free flying Anopheles stephensi mosquitoes. Southeast Asian J Trop Med Public Health 2005, 36:1423-1431.

65. Barnard DR: Repellency of essential oils to mosquitoes (Diptera: Culicidae). J Med Entomol 1999, 36:625-629.

66. Rutledge LC, Gupta L: Reanalysis of the C G Macnay Mosquito Repellent Data. J Vector Ecology 1995, 21:132-135.

67. Mondal S, Mirdha BR, Mahapatra SC: The science behind sacredness of Tulsi (Ocimum sanctum Linn.). Indian J Physiol Pharmacol 2009, 53:291-306.

68. Nzira L, Per M, Peter F, Claus B: Lippia javanica (Burm F) Spreng: its general constituents and bioactivity on mosquitoes. Trop Biomed 2009, 26:85-91.

69. Karunamoorthi K, llango K, Endale A: Ethnobotanical survey of knowledge and usage custom of traditional insect/mosquito repellent plants among the Ethiopian Oromo ethnic group. J Ethnopharmacol 2009, 125:224-229.

70. Kweka EJ, Mosha F, Lowassa A, Mahande AM, Kitau J, Matowo J, Mahande MJ, Massenga CP, Tenu F, Feston E, Lyatuu EE, Mboya MA Mndeme R, Chuwa G, Temu EA: Ethnobotanical study of some of mosquito repellent plants in north-eastern Tanzania. Malar J 2008, 7:152.

71. Gleiser RM, Bonino MA, Zygadlo JA: Repellence of essential oils of aromatic plants growing in Argentina against Aedes aegypti (Diptera: Culicidae). Parasitol Res 2010, DOl:10.1007/s00436-010-2042-4.

72. Misni N, Sulaiman S, Othman H, Omar B: Repellency of essential oil of Piper aduncum against Aedes albopictus in the laboratory. J Am Mosa Control Assoc 2009, 25:442-447.

73. Innocent $\mathrm{E}$, Joseph CC, Gikonyo NK, Nkunya MH, Hassanali A: Constituents of the essential oil of Suregada zanzibariensis leaves are repellent to the mosquito, Anopheles gambiae s.s. J Insect Sci 2010, 10:57.

74. USDA: Product Performance Test Guidelines. Insect Repellents for Human Skin and Outdoor Premises. Book Product Performance Test Guidelines. Insect Repellents for Human Skin and Outdoor Premises City; 1999.

75. Maguranyi SK, Webb CE, Mansfield S, Russell RC: Are commercially available essential oils from Australian native plants repellent to mosquitoes? J Am Mosq Control Assoc 2009, 25:292-300.

76. Tabanca N, Bernier UR, Tsikolia M, Becnel JJ, Sampson B, Werle C, Demirci B, Baser KH, Blythe EK, Pounders C, Wedge DE: Eupatorium capillifolium essential oil: chemical composition, antifungal activity, and insecticidal activity. Nat Prod Commun 2010, 5:1409-1415.

77. Thomas J, Webb CE, Narkowicz C, Jacobson GA, Peterson GM, Davies NW, Russell RC: Evaluation of repellent properties of volatile extracts from the Australian native plant Kunzea ambigua against Aedes aegypti (Diptera: Culcidae). J Med Entomol 2009, 46:1387-1391.

78. WHOPES: Guidelines for efficacy testing of mosquito repellents for human skin WHO/HTM/NTD/WHOPES/2009.4. Book Guidelines for efficacy testing of mosquito repellents for human skin WHO/HTM/NTD/WHOPES/2009.4 City: World Health Organisation; 2009.

79. Zhu JJ, Zeng XP, Berkebile D, Du HJ, Tong Y, Qian K: Efficacy and safety of catnip (Nepeta cataria) as a novel filth fly repellent. Med Vet Entomol 2009, 23:209-216.

80. Tuetun B, Choochote W, Kanjanapothi D, Rattanachanpichai E, Chaithong U, Chaiwong P, Jitpakdi A, Tippawangkosol P, Riyong D, Pitasawat B: Repellent properties of celery, Apium graveolens L., compared with commercial repellents, against mosquitoes under laboratory and field conditions. Trop Med Int Health 2005, 10:1190-1198.

81. Rutledge LC, Gupta RK: Variation in the protection periods of repellents on individual human subjects: an analytical review. J Am Mosa Control Assoc 1999, 15:348-355. 
82. Goodyer L, Behrens RH: Short report: The safety and toxicity of insect repellents. Am J Trop Med Hyg 1998, 59:323-324.

83. USEPA: Pesticide Registration Standard for $\mathrm{N}, \mathrm{N}$-diethyl-m-toluamide (DEET). Book Pesticide Registration Standard for N,N-diethyl-m-toluamide (DEET) City: Office of Pesticides and Toxic Substances Special Pesticides Review Division. United States Environmental Protection Agency; 1980.

84. Koren G, Matsui D, Bailey B: DEET-based insect repellents: safety implications for children and pregnant and lactating women. CMAJ 2003, 169:209-212.

85. Veltri JC, Osimitz TG, Bradford DC, Page BC: Retrospective analysis of calls to poison control centers resulting from exposure to the insect repellent $\mathrm{N}, \mathrm{N}$-diethyl-m-toluamide (DEET) from 1985-1989. J Toxicol Clin Toxicol 1994, 32:1-16.

86. Fradin MS: Mosquitoes and mosquito repellents: a clinician's guide. Ann Intern Med 1998, 128:931-940

87. USEPA: Reregistration Eligibility Decision (RED):DEET, EPA738-R-98-010. Book Reregistration Eligibility Decision (RED):DEET, EPA738-R-98-010 City: United States Environmental Protection Agency; 1998.

88. Sudakin DL, Trevathan WR: DEET: a review and update of safety and risk in the general population. J Toxicol Clin Toxicol 2003, 41:831-839.

89. McGready R, Hamilton KA, Simpson JA, Cho T, Luxemburger C, Edwards R, Looareesuwan S, White NJ, Nosten F, Lindsay SW: Safety of the insect repellent N,N-diethyl-M-toluamide (DEET) in pregnancy. Am J Trop Med Hyg 2001, 65:285-289.

90. Hinkle NCJ: Natural born killers. Pest Control Tech 1995, 23(7):54-57.

91. Duke JA, DuCellier JL: CRC Handbook of alternative cash crops. Boca Raton: CRC Press; 1993.

92. Zheng $\mathrm{H}$, He K: Intercropping in rubber plantations and its economic benefits. In Agroforestry Systems in China. Ottawa: International Development Research Centre (IDRC);IDRC 1993:

93. Greenstock DL, Larrea Q: Garlic as an insecticide. Book Garlic as an insecticide City: Doubleday Research Association; 1972, 12, pp. 12.

94. Nuchuchua O, Sakulku U, Uawongyart N, Puttipipatkhachorn S, Soottitantawat A, Ruktanonchai U: In vitro characterization and mosquito (Aedes aegypti) repellent activity of essential-oils-loaded nanoemulsions. AAPS PharmSciTech 2009, 10:1234-1242.

95. Moore SJ, Darling ST, Sihuincha M, Padilla N, Devine GJ: A low-cost repellent for malaria vectors in the Americas: results of two field trials in Guatemala and Peru. Malar J 2007, 6:101.

96. Choochote W, Chaithong U, Kamsuk K, Jitpakdi A, Tippawangkosol P, Tuetun B, Champakaew D, Pitasawat B: Repellent activity of selected essential oils against Aedes aegypti. Fitoterapia 2007, 78:359-364.

97. Trongtokit $Y$, Rongsriyam $Y$, Komalamisra N, Krisadaphong $P$, Apiwathnasorn C: Laboratory and field trial of developing medicinal local Thai plant products against four species of mosquito vectors. Southeast Asian J Trop Med Public Health 2004, 35:325-333.

98. Muller GC, Junnila A, Kravchenko VD, Revay EE, Butlers J, Schlein Y: Indoor protection against mosquito and sand fly bites: a comparison between citronella, linalool, and geraniol candles. J Am Mosa Control Assoc 2008, 24:150-153.

99. Ansari MA, Mittal PK, Razdan RK, Sreehari U: Larvicidal and mosquito repellent activities of Pine (Pinus longifolia, family: Pinaceae) oil. $J$ Vector Borne Dis 2005, 42:95-99.

100. Hao H, Wei J, Dai J, Du J: Host-seeking and blood-feeding behavior of Aedes albopictus (Diptera: Culicidae) exposed to vapors of geraniol, citral, citronellal, eugenol, or anisaldehyde. J Med Entomol 2008, 45:533-539.

101. Ritchie $S A$, Williams $C R$, Montgomery BL: Field evaluation of new mountain sandalwood mosquito sticks and new mountain sandalwood botanical repellent against mosquitoes in North Queensland, Australia. J Am Mosa Control Assoc 2006, 22:158-160.

102. Bernier UR, Furman KD, Kline DL, Allan SA, Barnard DR: Comparison of contact and spatial repellency of catnip oil and N,N-Diethyl-3methylbenzamide (Deet) Against Mosquitoes. J Med Entomol 2005, 42:306-311.

103. Noosidum A, Prabaripai A, Chareonviriyaphap T, Chandrapatya A: Excitorepellency properties of essential oils from Melaleuca leucadendron $\mathrm{L}$. Litsea cubeba (Lour.) Persoon, and Litsea salicifolia (Nees) on Aedes aegypti (L.) mosquitoes. J Vector Ecol 2008, 33:305-312.

104. Polsomboon S, Grieco JP, Achee NL, Chauhan KR, Tanasinchayakul S, Pothikasikorn J, Chareonviriyaphap T: Behavioral responses of catnip (Nepeta cataria) by two species of mosquitoes, Aedes aegypti and
Anopheles harrisoni, in Thailand. J Am Mosq Control Assoc 2008, 24:513-519.

105. Dell IT: Composition containing P-menthane-3, 8-diol and its use as an inset repellent. In Book Composition containing P-menthane-3, 8-diol and its use as an inset repellent. Volume 20100278755. City; 2010.

106. Corymbia citriodora.

107. Paluch G, Grodnitzky J, Bartholomay L, Coats J: Quantitative structureactivity relationship of botanical sesquiterpenes: spatial and contact repellency to the yellow fever mosquito, Aedes aegypti. J Agric Food Chem 2009, 57:7618-7625.

108. Waka M, Hopkins RJ, Glinwood R, Curtis C: The effect of repellents Ocimum forskolei and deet on the response of Anopheles stephensi to host odours. Med Vet Entomol 2006, 20:373-376.

109. Soderlund DM, Bloomquist JR: Neurotoxic actions of pyrethroid insecticides. Ann Rev Entomol 1989, 34:77-96.

110. Steketee RW, Campbell CC: Impact of national malaria control scale-up programmes in Africa: magnitude and attribution of effects. Malar J 2010, 9:299.

111. Cloyd RA: Natural indeed: are natural insecticides safer better than conventional insecticides? Illinois Pesticide Review 2004, 17: [http://www. pesticidesafety.uiuc.edu/newsletter/html/v17n304.pdf].

112. Govere J, Durrheim DN, Baker L, Hunt R, Coetzee M: Efficacy of three insect repellents against the malaria vector Anopheles arabiensis. Med Vet Entomol 2000, 14:441-444

113. Trigg JK: Evaluation of a eucalyptus-based repellent against Anopheles spp. in Tanzania. J Am Mosq Control Assoc 1996, 12:243-246.

114. Dugassa S, Medhin G, Balkew M, Seyoum A, Gebre-Michael T: Field investigation on the repellent activity of some aromatic plants by traditional means against Anopheles arabiensis and An. pharoensis (Diptera: Culicidae) around Koka, central Ethiopia. Acta Trop 2009, 112:38-42.

115. Seyoum A, Killeen GF, Kabiru EW, Knols BG, Hassanali A: Field efficacy of thermally expelled or live potted repellent plants against African malaria vectors in western Kenya. Trop Med Int Health 2003, 8:1005-1011.

116. Palsson K, Jaenson TG: Plant products used as mosquito repellents in Guinea Bissau, West Africa. Acta Trop 1999, 72:39-52.

117. Lukwa N, Per M, Peter F, Claus B: Lippia javanica (Burm F) Spreng: its general constituents and bioactivity on mosquitoes. Tropical biomedicine 2009, 26:85-91

118. Govere J, Durrheim DN, Du Toit N, Hunt RH, Coetzee M: Local plants as repellents against Anopheles arabiensis, in Mpumalanga Province, South Africa. Cent Afr J Med 2000, 46:213-216.

119. Seyoum A, Kabiru EW, Lwande W, Killeen GF, Hassanali A, Knols BG: Repellency of live potted plants against Anopheles gambiae from human baits in semi-field experimental huts. Am J Trop Med Hyg 2002, 67:191-195.

120. Dua VK, Gupta NC, Pandey AC, Sharma VP: Repellency of Lantana camara (Verbenaceae) flowers against Aedes mosquitoes. J Am Mosq Control Assoc 1996, 12:406-408.

121. White GB: The insect repellent value of Ocimum spp. (Labiatae): traditional anti-mosquito plants. East African Med J 1973, 50:248-252.

122. Waka M, Hopkins RJ, Curtis C: Ethnobotanical survey and testing of plants traditionally used against hematophagous insects in Eritrea. Journal of ethnopharmacology 2004, 95:95-101.

123. Park BS, Choi WS, Kim JH, Kim KH, Lee SE: Monoterpenes from thyme (Thymus vulgaris) potential mosquito repellents. Journal of the American Mosquito Control Association 2005, 21:80-83.

124. Ansari MA, Razdan RK: Repellent action of Cymbopogan martinii martinii Stapf var. sofia oil against mosquitoes. Indian J Malariol 1994, 31:95-102.

125. Karunamoorthi K, llango K, Murugan K: Laboratory evaluation of traditionally used plant-based insect repellent against the malaria vector Anopheles arabiensis Patton (Diptera: Culicidae). Parasitology research 2010, 106:1217-1223.

126. Sharma VP, Ansari MA: Personal protection from mosquitoes (Diptera: Culicidae) by burning neem oil in kerosene. J Med Entomol 1994, 31:505-507.

127. Tyagi BK, Ramnath T, Shahi AK: Evaluation of repellency of Tagetes minuta (Family:Compositae) against the vector mosquitoes Anopeles stephensi Liston, Culex quinquefasciatus Say and Aedes aegypti (L.). Int Pest Cont 1997, 39:184-185. 
128. Okoth J: Tagetes minuta $\mathrm{L}$. as a repellent and insecticide against adult mosquitoes. East African Med J 1973, 50:317-322.

129. El-Sheikh TM: Field evaluation of repellency effect of some plant extracts against mosquitoes in Egypt. J Egypt Soc Parasitol 2009, 39:59-72.

130. Lindsay SW, Janneh LM: Preliminary field trials of personal protection against mosquitoes in The Gambia using deet or permethrin in soap, compared with other methods. Med Vet Entomol 1989, 3:97-100.

doi:10.1186/1475-2875-10-S1-S11

Cite this article as: Maia and Moore: Plant-based insect repellents: a

review of their efficacy, development and testing. Malaria Journal 2011 10(Suppl 1):S11.

Submit your next manuscript to BioMed Central and take full advantage of:

- Convenient online submission

- Thorough peer review

- No space constraints or color figure charges

- Immediate publication on acceptance

- Inclusion in PubMed, CAS, Scopus and Google Scholar

- Research which is freely available for redistribution

Submit your manuscript at www.biomedcentral.com/submit 